\title{
RECHERCHES SUR L'INFLUENCE EXERCÉE PAR LES ALGINATES \\ DANS LA FABRICATION DES FROMAGES \\ A PATE MOLLE
}

\author{
par \\ Dr. Giovanni Delforno
}

\section{Introduction}

L'utilisation des algues marines par l'homme remonte à des époques très lointaines. On sait en effet que jadis les populations habitant au voisinage de la mer utilisaient les algues dans une mesure importante pour leur alimentation et celle des animaux domestiques. Les cendres des algues, très riches en sodium et en potassium, étaient aussi employées pour l'extraction de ces éléments et pour la fabrication du verre.

Pendant la première guerre mondiale, les algues ont été utilisées comme source de potasse, de brome, d'iode et - grâce à un processus de caractère fermentatif - d'acétone. Étant donné leur teneur importante en éléments minéraux, les algues ont été aussi étudiées attentivement depuis quelques dizaines d'années, en vue de leur utilisation comme fertilisants, mais cet emploi, quoique possible, n'a pas eu jusqu'à maintenant de développement pratique.

Des quantités considérables d'algues marines, séchées et réduites en poudre, sont aujourd'hui réservées à la production de farines qui, considérant leur teneur élevée en vitamines, facteur de croissance, en sels minéraux et en oligo-éléments reconnus essentiels pour les organismes vivants, entrent dans la composition des rations équilibrées modernes destinées à l'alimentation du bétail. Récemment, l'attention des chercheurs a été de nouveau tournée vers les algues marines, prenant en considération la possibilité d'en tirer profit sur une vaste échelle pour la production des alginates.

Les remarquables propriétés colloïdales et hydrophiles des alginates, jointes à la disponibilité de ces derniers, à leur constance de composition et à leurs multiples possibilités d'application, ont permis une rapide et toujours plus grande diffusion de leur emploi dans de nombreuses industries alimentaires, chimiques 
et pharmaceutiques comme agents épaississants, émulsionnants, gélifiants, de suspension, filmogènes et adhésifs. Dans de nombreux cas, ils représentent un produit de remplacement, sinon une amélioration, des gélatines animales, des pectines et des carragénines, des gommes du type adragante, acacia, karaya, etc. et des produits du type agar-agar et mousses d'Irlande.

"Algine » est la dénomination généralement utilisée pour désigner tant l'acide alginique (polymère de l'acide anhydro-dmannuronique) que ses dérivés - les alginates - dont les plus importants du point de vue industriel sont les alginates de sodium, de potassium, d'ammonium et de propylène-glycol.

L'algine est un polysaccharide colloïdal, lyophile, à structure chimique hautement complexe, qui est extrait de certaines espèces d'algues marines appartenant à la classe des Phéophycées et appelées communément algues brunes, à cause de leur couleur brune caractéristique, due à la présence d'un pigment spécial dit phéophylle. L'espèce dont proviennent les alginates pour la plus grande part est la Macrocystis pyrifera $\mathrm{Ag}$. ou " algue géante de l'Océan Pacifique "; des quantités mineures, cependant, sont extraites aussi d'autres espèces d'algues marines appartenant aux genres Fucus, Laminaria, Nerocystis, etc. que l'on trouve le long des côtes atlantiques des Etats-Unis d'Amérique, des Iles Britanniques et de l'Europe.

Pour de plus amples renseignements sur les caractères botaniques de ces algues, sur les méthodes d'extraction et sur les propriétés physiques et chimiques des produits alginiques, ainsi que sur leurs applications dans les diverses industries, nous renvoyons le lecteur à une publication précédente écrite sur ce sujet par l'auteur (Cf. DeLforno G.: Les alginates dans l'industrie laitière et fromagère. "Il Mondo del Latte ", Milan, XII, décembre $1958,817-822$.

Des expériences approfondies, exécutées in vivo sur des animaux de laboratoire, ont depuis un certain temps, mis en évidence le fait que les alginates pour l'usage alimentaire sont absolument sans aucune action toxique ou allergique, et c'est pourquoi ils peuvent trouver un emploi utile également dans la fabrication de certains produits dérivés du lait. En effet, par leur pouvoir exceptionnel d'épaissir la phase áqueuse et de stabiliser la lipophase, ils sont aujourd'hui largement utilisés dans la préparation des glaces alimentaires, de l'ice-cream, de la crème naturelle ou pasteurisée, du lait concentré, du yoghourt, d'entremets sucrés au lait et, en quantités mineures, dans la fabrication de fromages de table à pâte molle, de fromages fondus et de crèmes de fromage. 
La bibliographie et l'expérimentation relatives aux différentes applications des alginates dans l'industrie laitière et fromagère en Italie sont presque nulles, et en conséquence, pour combler au moins en partie ces lacunes, j'ai considéré utile d'entamer une série de recherches expérimentales sur l'influence exercée par ces colloïdes dans la fabrication de deux types de fromages à pâte molle piémontais, appelés Tomini et Robiole.

Tenant compte du fait qu'une amélioration de la technique de fabrication, en liaison avec une organisation commerciale plus adéquate, pourraient amener une augmentation graduelle de la production des dits fromages, on a conduit deux séries d'essais de fabrication de Tomini et de Robiole, un essai de contrôle et un essai expérimental, dans l'intention d'étudier principalement les modifications qui peuvent intervenir dans la composition chimique et dans les caractéristiques structurales des fromages préparés selon cette nouvelle méthodologie.

Au cours de l'exécution du présent travail, j'ai eu des renseignements vagues et indirects concernant des recherches effectuées surtout à l'étranger, sur cette question, mais, n'ayant pu retrouver la trace d'aucune de ces publications, je suis obligé de renoncer à toute référence bibliographique en la matière.

Avant de passer à l'exposé du plan des expériences effectuées et des conclusions que l'on a pu en tirer, qu'il me soit permis de remercier le Directeur de l'Agence Générale, pour l'Italie, de la Kelco Company de New-York, qui a encouragé et rendu possibles les recherches elles-mêmes et a offert, par le moyen de conseils, suggestions et assistance, la collaboration précieuse de ses techniciens.

\section{Partie expérimentale}

Les essais ont été conduits en appliquant des méthodes précises de recherche, en exécutant 40 fabrications de contrôle et autant de fabrications expérimentales de fromages Tomini et Robiole, dans quelques fromageries des provinces de Coni et de Turin particulièrement intéressées à la solution du problème.

Pour chaque fabrication, on a procédé à une répartition, exacte du lait en deux lots, à l'un desquels on a ajouté la solution alginique préparée selon les méthodes décrites plus loin, tandis que l'autre lot a servi de base de référence. La technique de fabrication suivie a été la même, également dans ses détails, aussi bien dans les fabrications expérimentales que dans les fabrications de contrôle. 
Au total, on a fabriqué 2868 Tomini et 520 Robiole qui, leur préparation terminée, ont été conservés dans des locaux convenables où ils ont été affinés rationnellement. Au bout de 2 à 9 jours pour les Tomini et au bout de 7 à 24 jours pour les Robiole, on a exécuté les contrôles du poids et les appréciations organoleptiques sur les fromages obtenus, opérations immédiatement suivies de déterminations d'ordre chimique.

Le type d'alginate, utilisé au cours des recherches, a été le produit appelé "Kelgin F ", qui est produit à l'échelle industrielle par la Kelco Company de New-York. Cet alginate est un hydrate de carbone colloïdal, soluble dans l'eau, d'une grande finesse (passant au tamis de 80 mailles au $\mathrm{cm}^{2}$ ), et de viscosité moyenne, hautement raffiné et comestible. Il se présente sous la forme d'une poudre granuleuse de couleur ivoire ou crème, transparente et très "coulante"; il est généralement inodore, mais il présente quelquefois une faible odeur de substance organique. Chimiquement, e'est le sel sodique d'un polymère de l'acide d-mannuronique.

Le "Kelgin F " est un produit de constitution constante et uniforme, qui est fabriqué sous un contrôle scientifique rigoureux et expérimenté avec le plus grand soin avant d'être livré dans le commerce, afin qu'il soit conforme aux spécifications y relatives. Il est aujourd'hui largement employé dans l'industrie laitière mondiale en raison de ses avantages spéciaux, qui en font un des meilleurs agents épaississants, émulsionnants, gélifiants, de suspension et stabilisants.

Ce type d'alginate est en mesure de se dissoudre complètement et assez rapidement, par simple agitation, tant dans l'eau chaude que dans l'eau froide, donnant une solution dense et uniforme essentiellement neutre $(p \mathrm{H}=7)$, qui ne coagule pas sous l'action de la chaleur, ni se gélifie sous l'action du froid; cette solution conserve inchangée sa particulière délicatesse dans une vaste gamme de températures, permettant ainsi à ceux qui l'utilisent de choisir le degré thermique répondant le mieux à leurs besoins, sans devoir procéder à un contrôle spécial de la température elle-même.

Le produit peut être dissous très facilement en l'ajoutant par petites doses successives à une certaine quantité d'eau et en agitant chaque fois assez énergiquement. Par le moyen de l'addition graduelle et de la forte agitation, on augmente considérablement la vitesse de dissolution de l'alginate et on diminue, en même temps, sa tendance à former des grumeaux, comme cela se passe par contre avec les colloïdes en poudres très fines et, d'une façon générale, avec toutes les gommes colloĭdales. 
de la couleur et de l'aspect de la croûte, de la structure, de la présence éventuelle de fissures, de points de ramollissement, de moisissures, etc. Chaque forme a été ensuite divisée en quatre parties égales, au moyen de deux tailles en croix, et examinée avec précision à l'intérieur ; cet examen a concerné surtout la couleur, l'aspect et la consistance de la pâte, la présence de trous ou de fentes, l'odeur et la saveur.

En ce qui concerne le prélèvement et la préparation des échantillons de fromages à analyser, j'ai respecté scrupuleusement toutes les normes relatives établies par la Commission internationale des fromages, de la Fédération internationale de laiterie. Pour éviter d'éventuelles erreurs dues à la perte d'eau que la substance examinée peut subir par évaporation, on a pris certaines précautions fondamentales parmi lesquelles celle de conserver les échantillons, déjà préparés pour l'analyse, dans des récipients de verre bien propres, secs, et munis de bouchons émeri, d'opérer avec le plus grand soin et d'exécuter toutes les opérations de pesée dans le temps le plus bref possible.

Au dosage de la matière grasse, nous avons fait précéder celui de l'eau et des substances volatiles à $95^{\circ} \mathrm{C}$. Aux fins de la présente recherche, cette donnée expérimentale revêtait un grand intérêt parce qu'elle a servi à établir des résultats exprimés en pourcentages d'extrait sec, sur la base desquels, comme on le sait, on doit émettre l'appréciation analytique légale de la teneur en matière grasse dans les fromages.

La détermination du pourcentage d'eau a été fait en opérant sur 4 à $6 \mathrm{~g}$ de fromage dans une capsule de porcelaine ou de verre à fond plat tarée, avec 20 à $30 \mathrm{~g}$ de poudre de quartz lavée et calcinée et avec une petite baguette en verre pour bien mélanger la masse, aussi bien avant qu'après les phases initiales de la dessiccation. Le récipient ainsi préparé a été introduit dans une étuve et soumis à la dessiccation à la température de $95^{\circ} \mathrm{C}$ pendant environ 7 heures, et cela jusqu'à obtenir un poids constant.

Sur le produit déshydraté, on a procédé ensuite à l'extraction de la matière grasse au moyen de l'appareil ordinaire à extraction continue de Soxhlet et selon le procédé décrit par E. SAvini. (E. SAVINI: Chimie et analyses du lait et des produits laitiers. Hoépli, Milan, 1946, II, 853-854.) La seule modification apportée à ce procédé a été le fait d'opérer sur 4 à $6 \mathrm{~g}$ de fromage au lieu de $2 \mathrm{~g}$, utilisant ainsi la même substance qui avait servi à la détermination de l'humidité.

Cette méthodologie, que l'on peut étendre aussi à d'autres produits, présente l'avantage de permettre une seule pesée de 
l'échantillon à examiner, évitant ainsi les inconvénients provenant de la difficulté de maintenir constant le degré d'humidité d'un échantillon qui doit être soumis aux deux déterminations analytiques. De cette façon, on simplifie la technique d'exécution et on garantit une plus grande précision en ce qui concerne la référence de la matière grasse au pourcentage d'extrait sec.

Commé méthode de dosage de la matière grasse renfermée dans le fromage, on a choisi la méthode pondérale par extraction à l'éther éthylique, exécutée directement sur le-produit desséché, car, à mon avis, ce procédé doit être considéré comme le plus sûr et le plus précis, celui qui justifie pleinement la grande confiance qui lui est accordée aujourd'hui par les chimistes et par les chercheurs dans leurs études intéressant le secteur fromager. De nombreuses recherches conduites à ce sujet, en effet, ont amplement démontré que la méthode "au sable» permet d'obtenir toute la matière grasse à son état primitif sans subir aucune altération, et qu'elle est en outre moins susceptible de conduire à des erreurs que n'importe quel autre procédé (y compris le procédé international de Schmid-Bondzynski-Ratzlaff), qui nécessite trop de manipulations avant de pouvoir obtenir l'extraction complète de la matière grasse hors de la masse du fromage.

\section{Résultats}

Lorsque la recherche a été terminée, les résultats ont été notés avec précision et attentivement étudiés afin de pouvoir en tirer des conclusions opportunes et définitives sur le sujet.

On a ainsi constaté, sur 20 échantillons de fromages Tomini, que la teneur moyenne en eau, présentée par l'ensemble des fromages témoins, était de 64,21 p. 100 , aveo des variations comprises entre une valeur minimale de 53,16 p. 100 et une valeur maximale de 72,77 p. 100 , tandis que le pourcentage d'eau, déterminé sur les fromages expérimentaux, ont été en moyenne de 65,17 p. 100 avec des oscillations entre un minimum de 52,98 p. 100 et un maximum de 74,59 p. 100 ; ces variations, assez considérables, doivent naturellement être mises en relation avec les différents degrés de maturation des produits examinés. L'extrait sec, obtenu par différence, présentait une valeur moyenne pour l'ensemble de 35,79 p. 100 dans la première série de fromages et de 34,83 p. 100 dans la seconde.

Les moyennes générales des pourcentages de matière grasse sur la substance humide étaient égales à 16,38 p. 100 (avec une valeur minimale de 12,22 p. 100 et une valeur maximale de 
21,50 p. 100) pour les Tomini de contrôle, et à 16,06 p. 100 (teneur minimale 12,19 p. 100 , teneur maximale 21,75 p. 100) pour les Tomini expérimentaux ; ces fortes oscillations sont manifestement dues à la richesse plus ou moins grande en matière grasse du lait de fromagerie et aux différents degrés de maturation atteints par les produits obtenus. Rapportée à la substance sèche, la matière grasse a été enregistrée à raison d'une teneur moyenne globale de 45,77 p. 100 (valeur minimale 42,96 p. 100 , valeur maximale 48,83 p. 100 ) dans le premier groupe de fabrications, et de 46,11 p. 100 (minimum 43,16 p. 100, maximum 49,04 p, 100) dans le second.

Sur la base de ces résultats analytiques, on peut donc observer que les Tomini préparés avec du lait additionné de "Kelgin F " présentaient en général des pourcentages d'humidité et de matière grasse sur extrait sec légèrement supérieurs à ceux qui sont observés dans les fromages pris comme termes de comparaison.

Les données rassemblées ont mis clairement en évidence que la différence constatée entre les teneurs moyennes en eau déterminées dans les fromages expérimentaux et dans les fromages témoins était de $+0,96 \mathrm{p}, 100$, avec des écarts variant entre une valeur maximale négative de $-0,41$ p. 100 et une valeur maximale positive de $+3,09$ p. 100 . Également, les pourcentages moyens totaux de matière grasse, rapportés au produit desséché, ont donné entre les deux séries de fabrications une différence moyenne positive égale à $+0,34$ p. 100 avec des écarts individuels oscillant entre une valeur maximale négative de $-0,44$ p. 100 et une valeur maximale positive de $+1,12$ p. 100 .

D'un autre côté, on a rassemblé les résultats relatifs aux analyses chimiques effectuées sur 20 échantillons de fromages Robiole produits selon la méthodologie normale, et sur un nombre égal d'échantillons de fromages du même type fabriqués avec du lait auquel on avait ajouté la solution alginique.

En examinant les données obtenues, on a pu observer que la teneur moyenne en eau présentée par l'ensemble des fromages témoins était de 53,06 p. 100 , avec des variations comprises entre une valeur minimale de 45,39 p. 100 et une valeur maximale de 65,31 p. 100, tandis que le pourcentage d'eau déterminé dans les fromages expérimentaux a été en moyenne de 53,80 p. 100 avec des oscillations entre un minimum de 45,15 p. 100 et un maximum de 67,48 p. 100. L'extrait sec obtenu par différence a présenté une valeur moyenne pour l'ensemble de 46,94 p. 100 dans la première série de fabrications et de 46,20 p. 100 dans la seconde. 
Les moyennes générales des pourcentages de matière grasse sur la substance humide ont été égales à 24,19 p. 100 (avec une valeur minimale de 16,27 p. 100 et une valeur maximale de 29,48 p. 100 ) dans les Robiole de contrôle et à 23,93 p. 100 (teneur minimale 15,36 p. 100 , teneur maximale 29,74 p. 100) dans les fromages expérimentaux; les fortes oscillations des teneurs en eau et en matière grasse enregistrées dans ces types de fromages doivent, elles aussi, être mises en relation avec les différentes teneurs en matière grasse du lait de fromagerie et avec le degré différent de maturation des produits analysés. Rapportée à l'extrait sec, la matière grasse a enregistré une teneur moyenne pour l'ensemble de 51,53 p. 100 (valeur minimale 45,49 p. 100 , valeur maximale 56,91 p. 100 ) dans le premier groupe de fabrication et de 51,80 p. 100 (minimum 46,09 p. 100, maximum 58,08 p. 100) dans le second.

Sur la base de cette seconde série de résultats analytiques, on peut donc noter que même dans les fromages Robiole, produits avec du lait contenant de l'alginate de sodium, on aurait en général des pourcentages d'humidité et de matière grasse sur l'extrait sec légèrement supérieurs à ceux qui sont rencontrés dans les fromages pris comme termes de comparaison.

Les données exposées, mettent bien en relief que la différence constatée entre les teneurs moyennes en eau déterminées dans les Robiole expérimentaux et dans les mêmes fromages témoins, a été de $+0,74$ p. 100 , avec des écarts variant entre une valeur maximale négative de $-0,31$ p. 100 et une valeur maximale positive de $+2,38$ p. 100. Également les pourcentages moyens totaux de matière grasse, référée au produit desséché, ont donné entre les deux séries de fabrications une différence moyenne positive égale à $+0,27$ p. 100 , avec des écarts individuels oscillant entre une valeur maximale négative de $-0,40$ p. 100 et une valeur maximale positive de $+1,17$ p. 100 .

\section{Conclusions}

De l'ensemble des recherches chimiques effectuées et aussi de l'ensemble des résultats positifs obtenus, nous croyons pouvoir déduire que l'utilisation des produits alginiques dans la fabrication des fromages de Tomino et de Robiola correspond très bien à l'objectif visé.

Les épreuves exécutées, en effet, ont permis de constater que l'emploi des alginates dans la préparation de ces fromages, a rendu possible d'obtenir des produits renfermant une plus forte teneur en matière grasse dans la substance sèche qui, comme on l'a dit, 
a été en moyenne de 0,34 p. 100 dans le groupe des Tomini et de 0,27 p. 100 dans celui des Robiole. Cette augmentation a trouvé une confirmation valable dans la réduction, plusieurs fois affirmée, de la dispersion de la matière grasse elle-même dans le sérum, et il y a lieu de considérer qu'elle est probablement due à une action de caractère physico-chimique exercée par le colloïde sur les globules gras de grand diamètre. Il convient de souligner à ce propos que, si cette action venait à être étudiée de façon plus approfondie, elle pourrait conduire à des développements d'intérêt théorique et pratique capitaux.

En ce qui concerne la teneur en matière grasse, on doit encore observer que 87,50 p. 100 du total des fromages expérimentaux produits ont permis d'enregistrer un écart positif par rapport aux fromages de contrôle, et il est aussi intéressant de noter que dans 5 de ces échantillons (c'est-à-dire 12,50 p. 100 du total), cette augmentation s'est montrée suffisante pour rendre les produits conformes, en ce qui concernait leur teneur en matière grasse, aux exigences fixées par les dispositions en vigueur de la législation fromagère italienne.

En ce qui concerne le poids des produits obtenus, il convient de faire remarquer que dans les groupes de fromages expérimentaux on rencontre un meilleur rendement en produits (évaluable en moyenne à raison de $1,5 \mathrm{~kg}$ de fromage par quintal de matière première à transformer) par rapport au rendement fourni par les fromages préparés selon la technique traditionnelle.

Les recherches entreprises ont mis encore en relief le fait que l'emploi du "Kèlgin F ", même à raison des proportions très faibles indiquées, permet d'obtenir des produits présentant une structure plus homogène et une consistance plus délicate, sans être affligés de la caractéristique préjudiciable de la gommosité. Cette délicatésse se conserve dans le temps et elle n'est en aucune façon altérée par les fluctuations de température qui interviennent pendant la période de stockage des produits. En outre, les alginates confèrent aux fromages un brillant et une élasticité particulièrement dignes d'être signalés et qui doivent sans doute s'inscrire parmi les caractères bénéfiques de ces colloïdes.

Les fromages expérimentaux produits ont été en général caractérisés par une pâte plus moelleuse et plus gélatineuse, d'un meilleur aspect, et dotée d'une crémosité plus grande, conservant en même temps la consistance désirée et une résistance plus élevée à la cassure. Ces fromages conservent inchangés aussi bien leur couleur que leur arôme typiques, présentant très souvent une 
saveur plus douce, agréable et raffinée, donc de nature à satisfaire les exigences actuelles du consommateur. En effet, ces fromages, qui ont été distribués à de nombreux consommateurs, à des experts et à des techniciens de l'industrie laitière et fromagère, hautement qualifiés, ont été très appréciés surtout en raison de leurs excellentes caractéristiques de délicatesse, d'homogénéité et de butyrosité de la pâte.

L'utilisation des alginates dans la fabrication des Tomini et des Robiole, non seulement rend les produits d'une qualité supérieure et d'une fabrication plus facile, mais aussi permet de faire enregistrer une notable réduction et dans certains cas une complète élimination de la séparation du sérum dans le produit fini, empêchant ainsi le produit lui-même de se durcir à l'extérieur et de devenir friable à l'intérieur pendant le stade de sa conservation. Ce fait a permis aussi de réduire et parfois d'éliminer le grave inconvénient du mouillage de l'emballage et de l'adhérence au matériau de conditionnement, qui est la cause principale des "rendus" pour cause d'acidification intérieure, de formation de croûte et de formation de moisissures.

Dans les fromages expérimentaux produits, en outre, on a remarqué presque toujours un plus grand volume que celui présenté par les fromages de contrôle. En particulier dans le groupe des Tomini, l'agent stabilisant a réussi à diminuer sensiblement le pouvoir de contraction de la masse de fromage, ce qui aurait nécessairement conduit à une diminution du volume de celui-ci, aux dépens du rendement, dans un produit pour lequel le volume revêt une importance notable : puisque, comme on le sait, il est généralement vendu à la pièce plutôt qu'au poids.

Après tout ce qui a été dit, on peut conclure que les résultats obtenus à la suite de ce travail expérimental ont été excellents, et il y a donc lieu de retenir que les alginates peuvent trouver une application de plus en plus vaste dans la préparation des différents types de fromages à pâte molle. Avant de terminer cette note, qui, je l'espère, pourra contribuer valablement à l'amélioration de la production des dits fromages, je voudrais m'adresser aux différents chercheurs et techniciens de l'industrie laitière et fromagère pour les exhorter à entreprendre et à développer convenablement les études et recherches également dans ce secteur de la technologie fromagère, afin de donner, en tenant compte du modernisme le plus poussé possible de l'aspect technique de la production, une impulsion nouvelle au progrès fromager auquel nous aspirons tous. 


\section{Summary}

It was acknowledged that you could get a better cheese yield and a better appearance in the making of soft cheeses (such as Tomino and Robiola) by adding half an ounce ( $15 \mathrm{~g})$ of alginates to 22 gallons (100 l) of milk.

So, it appears that the alginates can be beneficially used in the making of soft cheeses.

It seems désirable to carry out researches in that way.

\section{SUPPLÉMENT TECHNIQUE}

\section{RECHERCHES NEO-ZÉLANDAISES SUR LA FABRICATION DE LA CASÉINE SULFURIQUE par \\ G. GÉNIN \\ Ingénieur E.P.C.I.}

Avant 1952, date à partir de laquelle la fabrication de la caséine a diminué sensiblement aux États-Unis, à la suite de la mise en vigueur de la politique de soutien des prix des produits agricoles, la majeure partie de la caséine produite dans ce pays était obtenue par précipitation par l'acide sulfurique [1] du fait du bon marché de l'agent de précipitation. Deux procédés différents étaient utilisés : le procédé à la caillebotte pressée et le procédé à la caillebotte euite.

Dans le premier cas, la technique était très voisine de celle adoptée en Australie pour la préparation de la caséine chlorhydrique, on opérait toutefois à des températures et à des $p \mathrm{H}$ plus élevés, conditions conduisant à un caillé caoutchouteux, difficile à aciduler et à laver. Cependant, on avait pu obtenir industriellement des produits ne renfermant pas plus de 2,12 p. 100 de cendres.

Dans le procédé à la caillebotte cuite, on opérait à température et à $p \mathrm{H}$ encore plus élevés, conditions conduisant à un caillé se présentant sous la forme d'une masse cohérente, eneore plus difficile à laver et cela conduisant à une caséine à haute viscosité et à teneur élevée en cendres, dont les applications se trouvaient de ce fait limitées.

Ce sont ces constatations qui avaient conduit les spécialistes australiens et néo-zélandais à admettre que la caséine sulfurique 